\title{
THE MECHANICAL AND ELECTRIC CHARACTERISTICS OF TRANSMISSION LINES
}

\author{
BY HAROLD PENDER AND H. F. THOMSON
}

The object of this paper is to present in compact form the data and formulas, together with their derivations, required for the determination of the mechanical and electric characteristics of transmission lines, together with a set of charts whereby the various mathematical operations involved may be accomplished rapidly and with the minimum effort. The treatment naturally falls under two headings, A-Mechanical Characteristics, and B-Electric Characteristics. Summaries of the working symbols and equations for Part $A$ and Part $B$ follow the general discussion under these heads.

\section{A. Mechanical Characteristics}

The following discussion will be limited to the consideration of the characteristics of a perfectly flexible wire suspended from two fixed points of support. Actual wire spans closely approximate this condition, particularly when the successive spans are all of the same length, whether the supporting insulators be of the pin or of the suspension type. In the case of successive spans of unequal length the change in tension in the various spans, due to the variation of temperature and the mechanical load on the wire, will not be the same in each span and consequently such changes will produce a resultant force on the insulator, causing thereby a slight deflection of the top of the tower; or a deflection of the insulator only, if the latter is of the suspension type. This motion of the points of support will tend to equalize the tensions in the various spans. 
In the design of a wire span two problems must be solved: 1. At what tension (or sag) must the wire be strung in order that the tension in the span may not exceed a definite limit under the worst conditions of temperature and of mechanical loading (due to ice and wind)?; and 2 . What will be the maximum vertical sag of the wire for a given variation in loading and temperature? The factors which enter into these two problems are the following:

1. The length of the span.

2. The material of the wire.

3. The size of the wire.

4. The coefficient of linear expansion of the wire. For copper this coefficient is $9.6 \times 10^{-6}$ and for aluminum $12.8 \times 10^{-6}$, the temperature in each case being expressed in deg. fahr.

5 . The modulus of elasticity of the wire. The modulus of elasticity for copper or aluminum is not strictly a constant, but may be assumed as such as a first approximation. For copper this modulus varies from $12 \times 10^{6}$ to $16 \times 10^{6}$, depending upon the quality of the wire and upon whether the wire is stranded or solid. The former figure is generally used for stranded copper wires. For aluminum wire, which is always stranded when used for transmission lines, the modulus is equal to $9 \times 10^{6}$. The modulus $M$ is equal to the increase of tension $(\triangle T)$ in pounds per square inch required to produce an elongation $(\Delta \lambda)$ of a given length of wire $(\lambda)$ divided by the ratio of the elongation to the original length, i.e.,

$$
M=\Delta T \div \frac{\triangle \lambda}{\lambda}=\lambda \frac{\triangle T}{\triangle \lambda}
$$

6. The maximum tension in pounds per square inch to which the wire should be subjected. This maximum allowable tension is usually taken at one-half the ultimate tensile strength of the wire, about $30,000 \mathrm{lb}$. per sq. in. for copper and 13,000 1b. per sq. in. for aluminum.

7. The maximum external load to which the wire may be subjected due to the collection of sleet on the wire and the pressure of the wind against it. This, of course, will depend upon climatic conditions, but even for a given section of the country, there is considerable difference of opinion as to what should be assumed as a reasonable external load. The Joint Committee on Overhead Line Construction has recently recom- 
mended that the maximum loading for high-tension crossings be taken as that due to an ice coating 0.5 -in. thick all around the wire, plus a wind pressure of eight $1 \mathrm{~b}$. per sq. ft. of the projected area of this ice cylinder. To meet this requirement on a long span, especially with aluminum wire, would require a relatively large sag, from 30 to $100 \mathrm{ft}$., depending on the length of span and size of wire. This would require a prohibitive height of tower, at least for level country work. For cross country spans it would, therefore, seem advisable to assume a less heavy loading, particularly as an ice coating of 0.5 -in. and a wind velocity of 60 miles per hr. (corresponding approximately to eight $\mathrm{lb}$. wind pressure) will seldom, if ever, exist simultaneously.

8. The minimum temperature at which the maximum external load occurs. Here again there is considerable difference of opinion, even for given climatic conditions. The Joint Committee on Overhead Line Construction recommends the assumption of a minimum temperature of $-20 \mathrm{deg}$. fahr. for lines situated in the northern part of this country. For cross country spans the assumption of a higher temperature would seem more reasonable, in view of the fact that sleet seldom exists on a wire when the temperature is much below freezing.

9. The temperature at which the wire is to be strung. As an average stringing temperature $70 \mathrm{deg}$. fahr. is a reasonable assumption.

10. The maximum temperature to which the wire may be subjected. For lines located in the northern part of this country a maximum temperature of $120 \mathrm{deg}$. fahr. is reasonable. It should be noted that the temperature of a wire exposed to the sun is considerably higher than the temperature of the surrounding air.

The interrelation of these various quantities in the case of a wire suspended from two fixed points of support will be considered in detail.

\section{Determination of the Ratio $(K)$ of the Resultant Force ON THE Wire to THE Weight of the Wire}

The total force on a span of wire under any condition of mechanical loading consists in general of three components: 1, its own weight, 2 , the weight of the ice coating, and 3 , the force acting on it due to wind pressure. It is usual to assume that the wind pressure is horizontal, although it may have a considerable vertical component, particularly if the span is parallel to the 
base of a steep hill. The other two components of the resultant force on the wire are vertical.

Let $w=$ weight in pounds of one foot of the wire.

$d=$ diameter of wire in inches.

$i=$ thickness of ice coating in inches.

$v=$ wind pressure in pounds per square foot perpendicular to the wire.

$K=$ ratio of total force on the wire to weight of wire under these same conditions.

The vertical force in pounds on one foot length of the wire under a given mechanical load is then

$$
w+\frac{57}{144} \cdot \frac{\pi}{4}\left[(d+2 i)^{2}-d^{2}\right]=w+1.24 i(d+i)
$$

where the weight of ice is taken as $57 \mathrm{lb}$. per cu. ft. (Tests show that the skeet formed on a wire weighs less than this, due probably to air bubbles in it-this figure is, however, on the safe side.) The horizontal force acting on one foot of the wire is

$$
\frac{v}{12}(d+2 i)
$$

The resultant force acting on one foot of the wire is then

$$
\sqrt{(w+1.24 i(d+i))^{2}+\left(\frac{v(d+2 i)}{12}\right)^{2}}
$$

and therefore the value of the ratio $K$ is

$$
K=\sqrt{\left(1+\frac{1.24 i(d+i)}{w}\right)^{2}+\left(\frac{v(d+2 i)}{12 w}\right)^{2}}
$$

The values of this ratio $K$ for the various sizes of wire betwcen $1,000,000$ circular mils and No. 6, B. \& S. gage, for both copper and aluminum, for no ice and for ice thicknesses of $\frac{1}{4}, \frac{1}{2}$ and $\frac{3}{4}$ in., and for wind pressures from 0 to $12 \mathrm{lb}$. per sq. ft., are plotted on Chart No. 1. On this chart is also given a short table showing the relation between indicated wind velocity and pressure per square foot. The latter is deduced from the formula given by H. W. Buck in the Transactions of the International Electric 
Congress, 1904; this formula is that the wind pressure in pounds per square foot of projected area of a round cylinder of diameter $d_{1}$ produced by an actual wind velocity of $V$ miles per hour is $0.00021 V^{2} d_{1}$.

\section{The Equations of a Wire Span}

In the following discussion the wind pressure will be assumed perpendicular to the vertical plane through the two points of support and the pressure per foot of wire in the direction of the wind will be assumed constant at all points irrespective of the angle between the wire and the direction of the wind. This assumption is not strictly realized, since the wire near the points of support will make an angle of less than $90 \mathrm{deg}$. with the direction of the wind, and therefore to obtain an exact solution the wind pressure should be resolved into its two components, one acting in the direction of, and the other perpendicular to, the wind. Since the angle between the wire and the direction of the wire is in general very nearly 90 deg., the assumption that the wind pressure actually exerted on the wire is equal to the pressure which would be exerted were the wire in a vertical plane normal to the wind will not introduce an appreciable error. in comparison with the variations in the wind pressure which will occur due to the actual variation in wind velocity at different parts of the span.

The general case of the points of support at different elevations will also be considered, and the wire will be assumed to lie in a plane through the two points of support parallel to the direction of the resultant force. This assumption is not strictly realized when there exists a combination of wind pressure with the two points of support at different elevations, but is strictly true in all cases when the points of support are at the same elevation, and in the former case gives a solution as nearly correct as can be obtained without an elaborate analysis.

Fig. 1 is a diagram of a span of wire in perspective. This diagram shows the general case when the points of support are at different elevations and when there is a horizontal component of the force (wind pressure) acting on the wire. Fig. 2 shows only the plane $A E F B$, which is the plane in which the wire lies. When there is no wind this plane is vertical.

The plane $A E F B$ is determined by the line $A B$ and the direction of the resultant external force. The planes $A E R$ and $B F U$ are determined by the two components of the resultant 
force. $A G$ is drawn in the plane $A E F B$ through $A$ perpendicular to the resultant force. $E O F$ is drawn in the plane $A E F B$ perpendicular to the resultant force and through the point $O$ where the wire is also perpendicular to the resultant force.

Let $f=$ tension in pounds at any point $P$ of the wire.

$\alpha=$ angle between the tangent to the wire at this point and the line $A G$ drawn perpendicular to the resultant external force.

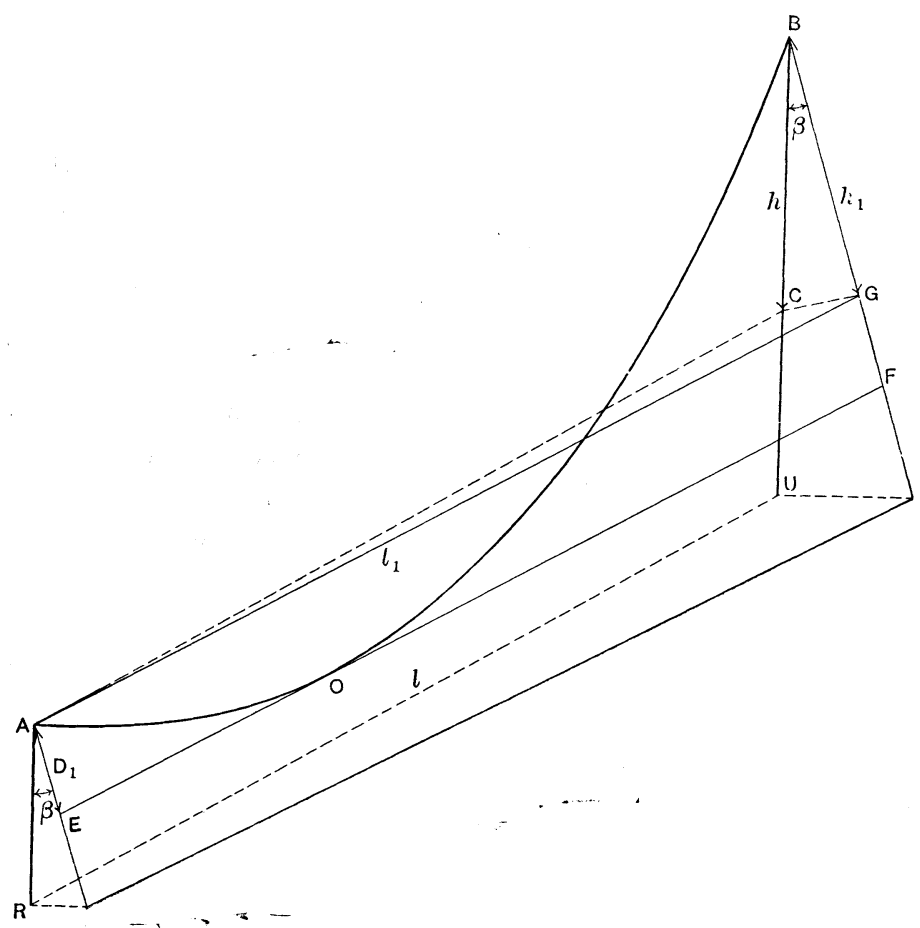

FIG. 1

$F=$ tension in pounds at the point $O$ where the wire is perpendicular to the resultant force.

$x=$ the horizontal distance between the point $P$ and the point $O$.

$y=$ perpendicular distance from $P$ to the line $O X$.

$\lambda=$ length of wire in feet from $O$ to $P$.

$d \lambda=$ an elementary length of the wire in feet at the point $P$. $K w=$ resultant force in pounds acting on a one-foot length of the wire due to its own weight, the weight of the ice coating and wind pressure. 
$m=$ weight in pounds of a bar of the conductor material one foot long and one square inch in cross section. (For copper $m=3.85$; for aluminum $m=1.16$ ).

$T=$ tension in wire in thousands of pounds per square inch at the point $O$.

Acting on the elementary length $d \lambda$ are three forces in the directions indicated. The resultant of these three forces must be zero. Hence, resolving these forces parallel to $O X$ and $O Y$ respectively, we have

$$
\begin{gathered}
(f+d f) \cos (\alpha+d \alpha)-f \cos \alpha=0 \\
(f+d f) \sin (\alpha+d \alpha)-f \sin \alpha-K w d \lambda=0
\end{gathered}
$$

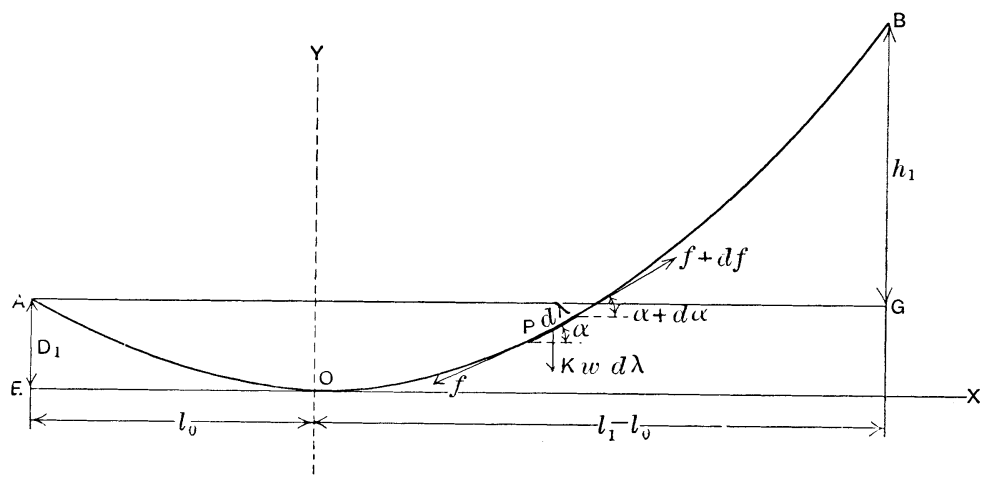

FIG. 2

But since $d \alpha$ is an infinitesimal angle its cosine is unity and its sine is equal to $d \alpha$. Hence,

$$
\begin{aligned}
& \cos (\alpha+d \alpha)=\cos \alpha-\sin \alpha d \alpha \\
& \sin (\alpha+d \alpha)=\sin \alpha+\cos \alpha d \alpha
\end{aligned}
$$

which, substituted in the above equations, give

$$
\cos \alpha d f-f \sin \alpha d \alpha=0
$$

$$
\sin \alpha d f+f \cos \alpha d \alpha=K w d \lambda
$$

which may in turn be written

$$
\begin{gathered}
d(f \cos \alpha)=0 \\
d(f \sin \alpha)=K w d \lambda
\end{gathered}
$$


Integrating these two equations, we gret

$$
f \cos \alpha=F
$$

and

$$
f \sin \alpha=K w \lambda
$$

Equation (4) shows that the component of the tension perpendicular to the resultant force is the same at all points of the wire. Equation (5) shows that the component of the tension at any point of the wire in the direction of the resultant force on the wire due to its own weight and external load is equal to the value of this force acting on that portion of the wire between this point and point $O$ at which the wire is perpendicular to the resultant force. The ratio of (5) and (4) gives

$$
\tan \alpha=\frac{K w \lambda}{F}
$$

This equation involves only the constants $K, w$ and $F$ and the angle $\alpha$ and distance $\lambda$; the two latter depend only on the shape of the curve in which the wire hangs. By substituting for $\alpha$ and $\lambda$ their values in terms of the coördinates $x$ and $y$, we can obtain the equation of this curve in rectangular coördinates. In (6) substitute $q=\tan \alpha$ and differentiate with respect to $x$; this gives

$$
\frac{d q}{\sqrt{ } 1+q^{2}}=\frac{K w}{F} d x
$$

The integral of this equation is*

$$
q=\sinh \frac{K w x}{F}
$$

*For any argument $u$ the hyperbolic sine is

$$
\sinh u=\frac{e^{u}-e^{-u}}{2}
$$

and the hyperbolic cosine is

$$
\cosh u=\frac{e^{u}+e^{-u}}{2}
$$

Chart No. 5 shows the relation between $u, \sinh u$, and $\cosh u$. 
Substitute for $q$ its value $\tan \alpha=\frac{d y}{d x}$ and integrate; this gives

$$
y=\frac{F}{K w}\left[\cosh \frac{K w x-1}{F}\right]
$$

This is the equation of the curve in which the wire hangs; it is the equation of the catenary.

The substitution in (6) of the value of $g=\tan \alpha$ given by (7) gives

$$
\lambda=\frac{F}{K w} \sinh \frac{K w x}{F}
$$

which is an expression for the length of wire $\lambda$ from $O$ to the point $P$ in terms of the corresponding distance $x$ measured along the line $O X$.

Since the weight $w$ of the wire per foot is equal to its cross section multiplied by the weight $m$ of a rod one foot long and one square inch in cross section, and since the tension in pounds $F$ is equal to the cross section of the wire multiplied by 1000 times the tension $T$ in thousands of pounds per square inch,

$$
\frac{w}{F}=\begin{gathered}
m \\
1000 T
\end{gathered}
$$

Hence equation (8) may be written

$$
y=\frac{1000 T}{K m}\left[\cosh \left(\frac{K m x}{1000 T}\right)-1\right]
$$

and equation (9) may be written

$$
\lambda=\frac{1000 T}{K m} \sinh \left(\begin{array}{c}
K m x \\
1000 T
\end{array}\right)
$$

The hyperbolic cosine of an argument $u$ may be expanded into the series

$$
\cosh u=1+\frac{u^{2}}{2 !}+\frac{u^{4}}{4 !}+\frac{u^{6}}{6 !}+\ldots \ldots \ldots
$$


and the hyperbolic sine into the series

$$
\sinh u=u+\frac{u^{3}}{3 !}+\frac{u^{5}}{5 !}+\frac{u^{7}}{7 !}+\ldots \ldots
$$

Therefore

$$
\begin{aligned}
\cosh u-1 & =\frac{u^{2}}{2}\left(1+\frac{u^{2}}{12}+\frac{u^{4}}{360}+\ldots \ldots\right) \\
\sinh u & =u\left(1+\frac{u^{2}}{6}+\frac{u^{4}}{120}+\ldots \ldots\right)
\end{aligned}
$$

Hence, for $u<0.48$, we may write, with an error of less than 2 per cent,

$$
\cosh u-1=\frac{u^{2}}{2}
$$

Also, with an error of less than 0.05 per cent,

$$
\sinh u=u\left(1+\frac{u^{2}}{6}\right)
$$

Hence for

$$
K m x<480 T
$$

equation (10) may be written, with an error of less than 2 per cent,

$$
y=\frac{K m x^{2}}{2000 T}
$$

which is the equation of a parabola. In all practical cases, except for extreme conditions of loading or for very long spans, the condition (12) is satisfied, and therefore for practical work the equation of a span of wire may be represented with sufficient accuracy by the parabola (13).

Under the same conditions, equation (11) for the length of wire between $O$ and $P$ may be written, with an error of less than 0.05 per cent,

$$
\lambda=x\left[1+\frac{1}{6}\left(\frac{K m x}{1000 T}\right)^{2}\right]
$$


But from (13)

$$
\frac{K m x}{1000 T}=\frac{2 y}{x}
$$

Therefore, to the same degree of accuracy,

$$
\lambda=x+\frac{2}{3} \quad \begin{array}{ll}
y^{2} \\
x
\end{array}
$$

\section{Deflection AND SAG.}

From the above equations the deflection and vertical sag of the wire for a given tension $T$ and loading factor $K$ may be deduced readily. In addition to the above symbols, let (see Figs. 1 and 2)

$l=$ length of span in feet; i.e., the horizontal distance between the points of support.

$l_{1}=$ distance $A G$ in feet.

$l_{0}=$ distance $E O$ in feet.

$D=$ deflection in feet of the point $O$ from the horizontal line through the two points of support when points of support are on the same level.

$p=\frac{100 \mathrm{D}}{l}=$ percentage deflection when points of support are on the same level.

$K^{\prime}=$ ratio of vertical component of external force to weight of wire ( $K^{\prime}$ is the value of $K$ when there is no wind).

$S=\frac{K^{\prime} D}{K}=$ vertical sag corresponding to the deflection $D$.

$h=$ difference in elevation in feet between the two points of support.

$h_{1}=$ the distance $B G$.

$D_{1}=$ deflection in feet of the point $O$ from the line $A G$ (Figs. 1 and 2.)

$S_{1}=$ vertical sag in feet corresponding to the deflection $D_{1}$.

From equation (13) and Fig. 2,

$$
y_{a}=D_{1}=\frac{K m l_{0}^{2}}{2000 T}
$$

and

$$
y_{b}=D_{1}+h_{1}=\frac{K m\left(l_{1}-l_{0}\right)}{2000 T}
$$


Subtracting we get

$$
h_{1}=\frac{K m}{2000 T}\left(l_{1}^{2}-2 l_{1} l_{0}\right)
$$

or

$$
l_{0}=\frac{l_{1}}{2}\left[1-\frac{2000 T h_{\imath}}{K m l_{1}^{2}}\right]
$$

The relations between $l_{1}$ and $l$ and between $h_{1}$ and $h$ may be deduced from Fig. 1 . The plane $B C G$ is perpendicular to $A C$ and therefore perpendicular to the plane $A C G$. Also the angle $B G A$ is a right angle. Hence the angle $B G C$ is a right angle. Therefore

$$
h_{1}=h \cos \beta=\frac{K_{1} h}{K}
$$

Also, $A C$ is perpendicular to $G C$, since $A C$ is perpendicular to the plane $B \min$; therefore

$$
l_{1}=\sqrt{l^{2}}+h^{2} \sin ^{2} \beta=l \sqrt{1+\left(\frac{h \sin \beta}{l}\right)^{2}}
$$

The ratio of $h$ to $l$ in any practical case will not exceed 0.25 and the angle $\beta$ will not exceed $45 \mathrm{deg}$. Hence $l_{1}$ will differ from $l$ by less than 2 per cent. As a first approximation then we may write equation (16)

$$
l_{0}=\frac{l}{2}\left[1-\frac{2000 T K^{\prime} h}{K^{2} m l^{2}}\right]
$$

For $h=0$, that is, for the points of support on the same level, $l_{0}=\frac{l}{2}$; the point $O$ is then at the center of the span. Hence, for points of support at the same level,

$$
D=\frac{K m l^{2}}{8000 T}
$$

The percentage deflection is then

$$
p=\frac{K m l}{80 T}
$$


Chart No. 2 gives the value of this percentage deflection for both copper and aluminum for the various values of $(K l)$ and $T$ which are likely to arise in practise. The vertical sag corresponding to $D$ is

$$
S=\frac{K^{\prime} D}{K}
$$

For a difference of elevation $h$ we then have, from (16a) and (17),

$$
l_{0}=\frac{l}{2}\left[1-\begin{array}{cc}
K^{\prime} & h \\
4 K & K
\end{array}\right]
$$

$D$ is the deflection corresponding to the same length of span, same tension, and same loading but the two points of support at the same elevation. Substituting (19) in (15) gives

$$
D_{1}=D\left(1-\frac{K^{\prime} h}{4 K D}\right)^{2}
$$

and the vertical sag corresponding to $D_{1}$ will not exceed

$$
S_{1}=\frac{K^{\prime} D_{1}}{K}
$$

(An inspection of Fig. 1 will show that the vertical sag may be slightly less than this; formula (21), however, is on the safe side.)

From equation (19) it is seen that the distance of the point $O$ to the lower point of support becomes negative when

$$
h<\frac{4 K D}{K^{\prime}}
$$

This means that when $h<\begin{gathered}4 K D \\ K^{\prime}\end{gathered}$ the point $O$ lies outside of the span; i.e., the wire has a continuous upward slope from the lower to the higher support. This condition is illustrated in Fig. 3. Since the vertical force downward on the insulator due to the wire between $A$ and $B$ is equal to $K^{\prime} w l_{0}$, if $l_{0}$ is negative there will be an upward pull at $A$.

If this force is greater than the downward pull due to the span on the other side of this support, the resultant force on the 
insulator will be upward. In laying out a line this condition should of course be avoided by relocating the poles.

When the point $O$ coincides with the lower point of support, $l_{0}=0$, and the horizontal distance between $O$ and the higher point of support is equal to the length of the span $l$. Hence the greatest value of $\frac{K m x}{1000 T}$ (see equations 10 and 12 ) for any point in the span is equal to $\frac{K m l}{1000 T}$ and this in turn is equal to $\frac{8 p}{100}$ where $p$ is the per cent deflection which would be produced by the same tension and loading were the points of support on

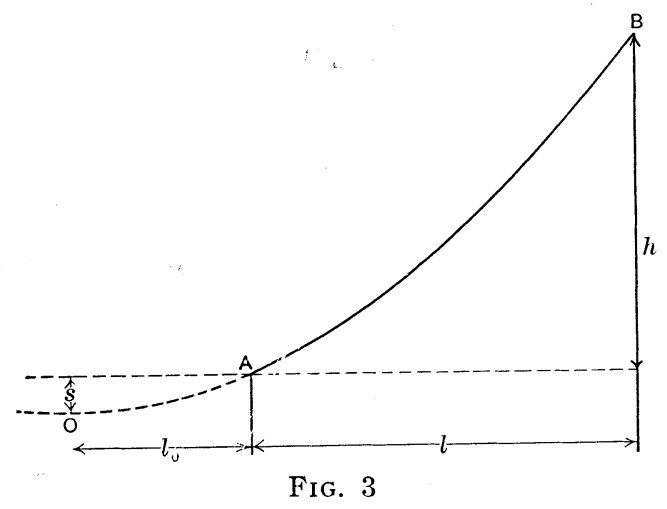

the same level (see equation 18). Hence the condition that the approximate equations (13) and (14) may be applied is that

$$
p<6 \text { per cent }
$$

Hence the maximum error which can arise from the use of Chart No. 2 for the determination of deflection and sag is less than 2 per cent.

\section{EfFEct of Variations in Temperature and Mechanicai. LOADING}

We wish next to determine how the tension and deflection of a span of wire varies when the temperature of the wire changes and when the mechanical load on the wire changes (due to the formation or melting of the sleet and changes in wind pressure.) We shall assume that the points of support remain rigidly fixed. 
Let

$t=$ temperature in deg. fahr. under any given set of conditions.

$T=$ tension at $O$ in thousands of pounds per square inch under these same conditions.

$K=$ ratio of resultant force on wire to weight of wire under these same conditions.

$K^{\prime}=$ ratio of vertical component of resultant force to weight of wire under these same conditions.

$\lambda_{1}=$ length of the wire in feet under these same conditions. $t_{0}, T_{0}, K_{0}, K_{0}{ }^{\prime}, \lambda_{0}$ designate the same quantities for any other set of conditions.

$a=$ coefficient of linear expansion of the wire per deg. fahr.

$M=$ modulus of elasticity of the wire in (pounds per square inch) units.

Other symbols as above.

From equations (14) and (15) and Figs. 1 and 2 we have that the length of the wire in the span under any conditions of temperature and loading is

$$
\begin{aligned}
\lambda_{1} & =l_{0}+\frac{2}{3} \frac{D_{1}^{2}}{l_{0}}+l-l_{0}+\frac{2}{3} \frac{\left(D_{1}+h_{1}\right)^{2}}{l-l_{0}} \\
& =l+\frac{2}{3}\left(\frac{K m}{2000 T}\right)^{2}\left[l_{0}^{3}+\left(l-l_{0}\right)^{3}\right] \\
& =l+\frac{2}{3}\left(\frac{K m}{2000 T}\right)^{2}\left[l^{3}-3 l l_{0}\left(l-l_{0}\right)\right]
\end{aligned}
$$

From equation (16a)

$$
\begin{aligned}
& l_{0}=\frac{l}{2}\left[1-\frac{2000 T h K^{\prime}}{K^{2} m l^{2}}\right] \\
& l-l_{0}=\frac{l}{2}\left[1+\frac{2000 T h K^{\prime}}{K^{2} m l^{2}}\right]
\end{aligned}
$$

whence

$$
\dot{\lambda_{1}}=l+\frac{2}{3}\left(\frac{K m}{2000 T}\right)^{2} l^{3}\left[\frac{1}{4}+\frac{3}{4}\left(\begin{array}{c}
2000 T h K^{\prime} \\
K^{2} m l^{2}
\end{array}\right)^{2}\right]
$$


or

$$
\lambda_{1}=l\left[1+\frac{1}{6}\left(\frac{K m l}{2000 T}\right)^{2}+\frac{1}{2}\left(\frac{K^{\prime} h}{K l}\right)^{2}\right]
$$

From (18)

$$
\frac{K m l}{2000 T}=\frac{. p}{25}
$$

where $p$ is the per cent deflection for the same condition of temperature, loading and tension, but for points of support on the same level. Hence the expression for $\lambda_{1}$ may be written

$$
\lambda_{1}=l\left[1+\frac{p^{2}}{3750}+\frac{1}{2}\left(\begin{array}{cc}
K^{\prime} & h \\
K & l
\end{array}\right)^{2}\right]
$$

Similarly, for any other conditions of temperature and loading the length of the wire is

$$
\lambda_{0}=l\left[1+\frac{p_{0}^{2}}{3750}+\frac{1}{2}\left(\frac{K_{0}^{\prime} h}{K_{0} l}\right)^{2}\right]
$$

Hence the change of length due to the change in temperature and loading is

$$
\lambda_{1}-\lambda_{0}=l\left[\frac{p^{2}-p_{0}^{2}}{3750}+\frac{1}{2}\left(\frac{K^{\prime} h}{K l}\right)^{2}-\frac{1}{2}\left(\frac{K_{0}^{\prime} h}{K_{0} l}\right)^{2}\right]
$$

If the points of support remain fixed this change of length can result only from a stretching (or contraction) of the wire due to change in tension and to an expansion (or contraction) of the wire due to change in temperature. This change in length may, therefore, also be expressed in terms of the temperature coefficient $a$, the difference of temperature $t-t_{0}$, the modulus of elasticity $M$, and the change in the resultant tension in the wire.

The resultant tension in the wire, at any point a distance $\lambda$ measured along the wire from the point $O$, is equal to

$$
T^{\prime}=\sqrt{f_{0}^{2}+(K w \lambda)^{2}}=T \sqrt{1+\left(\frac{K m \lambda}{1000 T}\right)^{2}}
$$

See equations (4) and (5). 
The maximum value of $T^{\prime}$ for a given value of $T$ will occur at the higher point of support, i.e., for

$$
\lambda=l-l_{0}=\frac{i}{2}\left[1+\frac{K^{\prime} h}{4 K D}\right]
$$

See Fig. 1 and equation (19). Also, from equation (18)

$$
\frac{K m l}{2000 T}=\frac{p}{25}
$$

whence the maximum value of $T^{\prime}$ is

$$
T^{\prime}=T \sqrt{1+\frac{p^{2}}{625}}\left(1+\begin{array}{c}
K^{\prime} h \\
4 K \frac{h}{D}
\end{array}\right)^{2}
$$

The maximum value of $p$ likely to arise in practise is 6 per cent, and the maximum value of $h$ is $\frac{4 K D}{K}$ (lowest point of the wire coinciding with lower point of support), and hence the maximum value of $T^{\prime}$ likely to arise in practise is

$$
T^{\prime}=1.11 T
$$

The limiting case of a 6 per cent deflection rarely occurs, and even in this case the resultant tension is equal to the higher value, $1.11 T$ only at the higher point of support; for the greater part of the length of the wire the resultant tension is sensibly equal to the tension $T$ at the point $O$. Hence in calculating the stretching of the wire by the resultant tension we may take, as a close approximation, the resultant tension at every point equal to the tension at $O$.

The change in length due to a change of temperature from $t_{0}$ to $t$ is

$$
a\left(t-t_{0}\right) \lambda_{1}
$$

and the change in length due to a change in tension from $T_{0}$ to $T$ is

$$
\frac{1000\left(T-T_{0}\right) \lambda_{1}\left[1+a\left(t-t_{0}\right)\right]}{M}
$$

But since the coefficient of expansion $a$ is a very small quantity (of the order of $\left.10^{-5}\right)$ the term $a\left(t-t_{0}\right)$ may be neglected in 
comparison with unity for any value of $t-t_{0}$ likely to arise in practise $\left(t-t_{0}\right.$ will never be greater than $150 \mathrm{deg}$. fahr.). Hence the total change in length $\left(\lambda_{1}-\lambda_{0}\right)$ may be written

$$
\lambda_{1}-\lambda_{0}=\left[a\left(t-t_{0}\right)+\frac{1000\left(T-T_{0}\right)}{M}\right] \lambda_{1}
$$

Again, for $p<6$ per cent, $\lambda_{1}$ will differ from $l$ by less than one per cent (see equation 23); hence, with an error of less than one per cent,

$$
\lambda_{1}-\lambda_{0}=\left[a\left(t-t_{0}\right)+\frac{1000\left(T-T_{0}\right)}{M}\right] l
$$

Equating (23) and (25) we have

$$
\begin{aligned}
& \left(p^{2}-\frac{3.75 \times 10^{6} T}{M}\right)-\left(p_{0}{ }^{2}-\frac{3.75 \times 10^{6} T_{0}}{M}\right)= \\
& 3750 a\left\{\left(t-t_{0}\right)-\frac{h^{2}}{2 a l^{2}}\left[\left(\frac{K^{\prime}}{K}\right)^{2}-\left(\frac{K_{0}^{\prime}}{K_{0}}\right)^{2}\right]\right\}
\end{aligned}
$$

For the points of support on the same level $(h=0)$ or for no change in the position of the plane of the $\operatorname{span}\left(\frac{K_{0}{ }^{\prime}}{K_{0}}=\frac{K^{\prime}}{K}\right.$; a particular instance of which is no wind pressure, in which case the plane of the span remains vertical), this equation reduces to

$$
\left(p^{2}-\frac{3.75 \times 10^{6} T}{M}\right)-\left(p_{0}^{2}-\frac{3.75 \times 10^{6} T_{0}}{M}\right)=3750 a\left(t-t_{0}\right)
$$

Hence, when the tension $T_{0}$ at temperature $t_{0}$ and the loading factor $K_{0}$ are known, the tension $T$ at any other temperature $t$ and loading factor $K$ may be determined by solving this equation for $T$, after substituting for $p$ and $p_{0}$ their values

$$
p=\frac{K m l}{80 T} \text { and } p_{0}=\frac{K_{0} m l}{80 T_{0}}
$$

This equation, however, is a cubic in $T$, and its solution by algebraic methods is extremely difficult. By making use of Chart 
No. 2, however, its solution may be obtained without any arithmetical computations whatever other than a simple multiplication.*

The curved lines on this chart give $p^{2}$ as ordinates (actual vertical distances equal $p^{2}$; the scale on the left of the chart gives $p$, which is the square root of the vertical distances) against $T$ as abscissas. The numbers on the curves give the corresponding value of $(K l)$. Two scales for the tension $T$ are given, one for copper and one for aluminum, this arrangement making possible the use of the same set of curves for both materials. The three heavy straight lines at the base of the chart are drawn so that the ordinate (actual distance) of any point on any one of these lines is equal to

\section{$3.75 \times 10^{6} \mathrm{~T}$ $M$}

where $T$ is the abscissa of this point on the proper tension scale and $M$ is the modulus of elasticity of the wire. $\dagger$

The small vertical scales in the lower left hand corner of the chart marked "Temperature Correction " are laid off so that the vertical distance between any two temperature marks $t$ and $t_{0}$ is equal to

$$
3750 a\left(t-t_{0}\right)
$$

\section{SUMMARY}

The method of solving equation (27) by means of Chart No. 2 is given below. First, however, a summary of the working symbols will be convenient:

$l=$ length of span in feet.

$h=$ difference in elevation of points of support in feet.

*The physical constants for copper-clad steel wire whose conductivity is 40 per cent of that for the same size of copper wire are:

$a=6.7 \times 10^{-6}$ per deg. fahr.; $M=22 \times 10^{6} \mathrm{lb}$. per square in.; $m=$ $3.58 \mathrm{lb}$. per ft. The additions to Chart No. 2 for operating with this wire are as follows:

1. Tension scale equal to 0.93 of copper tension scal a per in.

2. Modulus guide line through the origin and the point $p=2.8$, $T_{\text {copper }}=50$.

3. Temperature correction scale equal to 1.43 of that per in. for copper.

$\dagger$ A serious objection to another graphical solution for calculations of this sort which was published by one of the authors in the Electrical World, Sept. 28,1907 , was the necessity of a separate guide line for every length of span. This difficulty has been obviated in the above method. The angular location of the tension axis in the present form of chart was suggested by Mr. R. S. Brown of Telluride, Colo. 
$T_{0}=$ the tension at the point $O$ (Figs. 1 and 2) in thousands of pounds per square inch under any given condition of temperature and loading.

$t_{0}=$ temperature in deg. fahr. when the tension is $T_{0}$.

$K_{0}=$ ratio of resultant force due to weight of wire, weight of ice and wind pressure, to weight of wire (Chart No. 1) when the tension is $T_{0}$.

$K_{0}{ }^{\prime}=$ ratio of the vertical component of this force to weight of wire when the tension is $T\left(K^{\prime}{ }_{0}=\right.$ value of $K$ from Chart No. 1 corresponding to the given size of wire and the given thickness of ice, but no wind.)

$T=$ tension in thousands of pounds per square inch for any other condition of temperature and loading.

$t=$ temperature in deg. fahr. when the tension is $T$.

$K=$ ratio of resultant force due to weight of wire, weight of ice and wind pressure, to weight of wire when the tension is $T$. (For no ice and no wind $K=1$.)

$K^{\prime}=$ ratio of the vertical component of this force to weight of wire when tension is $T$. (For no ice and no wind $\left.K^{\prime}=1\right)$.

$D=$ deflection in feet of the lowest point of the wire from the line through the points of support when the tension is $T$ and the points of support are on the same level. $D$ is measured in the plane of the curve in which the wire hangs.

$p=\frac{100 D}{l}=$ per cent deflection corresponding to $D$.

$S=$ vertical sag in feet of the lowest point of wire below the points of support when the tension is $T$ and the points of support are on the same level.

$D_{1}=$ deflection in feet of the point $O$ from the line $A G$ (see Figs. 1 and 2) when the tension is $T$ and the points of support are not on the same level. (For $h=$ $\frac{4 K D}{K^{\prime}}$ the lower point of support is the lowest point of the wire.)

$S_{1}=$ vertical sag in feet of the lowest point of the wire below the lower point of support.

$a=$ temperature coefficient of wire when temperature is expressed in deg. fahr. (for copper $a=9.6 \times 10^{-6}$, for aluminum $\left.a=12.8 \times 10^{-6}\right)$. 
To find the tension $T$ and the corresponding deflection and sag when $l, h, T_{0}, t_{0}, K_{0}, K_{0}{ }^{\prime}, t, K$ and $K^{\prime}$ are given, Chart No. 2 is used as follows, when $\frac{K^{\prime}}{K}=\frac{K_{0}{ }^{\prime}}{K_{0}}$ :

1. On a straight strip of paper mark a reference point $A$ and vertically above this point mark a second point $B$ such that the distance $A B$ is equal to $t-t_{0}$ measured on the temperature scale.

2. Next place the strip of paper with its edge along the ordinate passing through the tension $T_{0}$ and make the point $A$ coincide with the intersection of the edge of the strip and the curve marked with the number equal to $\left(K_{0} l\right)$. Mark on the strip the point $C$ where the heavy straight line marked with the proper modulus $M$ intersects the edge of the strip.

3. Keep the strip of paper vertical and slide the point $C$ along the modulus line until the edge of the strip at $B$ intersects the curve marked with the number equal to $(K l)$. The abscissa of the point $B$ is then equal to the tension $T$ corresponding to the temperature $t$ and loading factor $K$.

The ordinate of the point $B$ (read on the vertical scale on the left) is the per cent deflection $p$ corresponding to this tension $T$ and loading factor $K$ when the points of support are on the same level. Then the corresponding deflection in feet is

$$
D=\frac{p l}{100}
$$

and the vertical sag is

$$
S=\frac{K^{\prime} D}{K}
$$

If the difference in elevation of the two points of support is $h$, the deflection of the lowest point of the wire is

$$
D_{1}=D\left(1-\frac{h K^{\prime}}{4 K D}\right)^{2}
$$

and the vertical deflection is

$$
S_{1}=S\left(1-\frac{h K^{\prime}}{4 K D}\right)^{2}
$$


The deflection and sag corresponding to $T_{0}$ are calculated in exactly the same manner from the per cent deflection $p_{0}$ corresponding to the tension $T_{0}$ and the loading factor $K_{0}$; whether the sag corresponding to $T$ will be greater than that corresponding to $T_{0}$ will depend upon the temperatures and loading factors.

In case the points of support are not on the same level and there is a change in the direction of the plane of the wire, (i.e., $\left.\frac{K^{\prime}}{K} \neq \frac{K_{0}^{\prime}}{K_{0}}\right)$, the new tension $T$ must be found by solving equation (26). This can also be done by means of Chart No. 2, for the term

$$
-\frac{h}{2 a l^{2}}\left[\left(\frac{K^{\prime}}{K}\right)^{2}-\left(\frac{K_{0}^{\prime}}{K_{0}}\right)^{2}\right]
$$

is equivalent to a decrease of temperature numerically equal to this expression. Hence the only change in the procedure described above is to consider the temperature rise not as $t-t_{0}$, but as

$$
\left(t-t_{0}\right)-\frac{h^{2}}{2 a l^{2}}\left[\left(\frac{K^{\prime}}{K}\right)^{2}-\left(\frac{K_{0}{ }^{\prime}}{K_{0}}\right)^{2}\right]
$$

The rest of the procedure is exactly the same as before. This correction due to the difference between $\frac{K_{0}{ }^{\prime}}{K_{0}}$ and $\frac{K^{\prime}}{K}$ may be important, particularly in passing from the condition of no ice and no wind to the condition of ice and wind, for in the latter case $\left(\frac{K_{0}{ }^{\prime}}{K_{0}}\right)^{2}$ may be as great as $\frac{1}{2}(45 \mathrm{deg}$. deflection of the plane of the wire from the vertical) whereas for the former $\frac{K^{\prime}}{K}=1$.

Similarly, in the reverse problem of passing from the condition of ice and wind to the condition of no ice and no wind there will be a like correction, but the formula without this correction factor will give results on the safe side.

It should also be noted that the maximum resultant tension in the span under any condition of temperature and loading is not equal to the tension $T$ at the point $O$ but in general is equal to

$$
T^{\prime}=T \sqrt{1+\frac{p^{2}}{625}\left(1+\frac{h K^{\prime}}{4 K D}\right)^{2}}
$$


(see equation 24). Similarly, the maximum resultant tension under the "zero" conditions is

$$
T_{0}^{\prime}=T_{0} \sqrt{1+\frac{p_{0}^{2}}{625}\left(1+\frac{h K_{0}^{\prime}}{4 K_{0} D_{0}}\right)^{2}}
$$

\section{EXAMPLE}

Given a No. 0 B \& S aluminum wire; $l=200 \mathrm{ft}$.; $T_{0}=13$; $\left(t-t_{0}\right)=70 \mathrm{deg}$. fahr. for $\frac{1}{2} \mathrm{in}$. of ice and $8 \mathrm{lb}$. per sq. ft. of wind $K_{0}=11.6 ; K_{0}{ }^{\prime}=6.7$; for no ice nor wind $K=K^{\prime}=1$.

Then

$$
h=0.0 \mathrm{ft} . \quad h=10.0 \mathrm{ft} .
$$

$$
\begin{aligned}
& \left(t-t_{0}\right)-\frac{h^{2}}{2 a l^{2}}\left[\left(\frac{K^{\prime}}{K}\right)^{2}-\left(\frac{K_{0}^{\prime}}{K_{0}}\right)^{2}\right] \quad 70 \quad 4.9 \\
& K_{0} l \ldots \ldots \ldots \ldots \ldots \ldots 2320 \quad 2320 \\
& K l \ldots \ldots \ldots \ldots \ldots \ldots 200 \\
& \begin{array}{l}
P_{0} \ldots \ldots \ldots \ldots \ldots \ldots \\
\hline
\end{array} \\
& D_{0} \ldots \ldots \ldots \ldots \ldots \ldots . \quad 5.16 \mathrm{ft} . \quad 2.68 \mathrm{ft} \text {. } \\
& S_{0} \ldots \ldots \ldots \ldots \ldots \ldots \ldots \text {. } 2.98 \mathrm{ft} . \quad 1.55 \mathrm{ft} \text {. } \\
& \text { T.............. } 1.28 \text { thou- } 1.92 \text { thou- } \\
& \text { sand } 1 \mathrm{~b} \text {. sand } 1 \mathrm{~b} \text {. } \\
& \text { p.............. } 2.27 \% \quad 0.49 \% \\
& D \ldots \ldots \ldots \ldots \ldots \ldots \ldots \quad 4.54 \mathrm{ft} . \quad 0.9 \mathrm{ft} \text {. } \\
& S \ldots \ldots \ldots \ldots \ldots \ldots f \mathrm{ft} . \quad 0.96 \ldots
\end{aligned}
$$

\section{B. Electric Characteristics}

When a given amount of electric power is transmitted over a transmission line to a substation or other receiver, a certain amount of power is lost in the line. There is in general a difference in the voltage between wires at the two ends of the line, and the power factor at the sending and receiving ends will be different. The power loss, voltage loss, and change in powe: factor in general depend upon the following factors:

1. The amount of power delivered.

2. The voltage at which the power is delivered.

3. The power factor at the receiving end.

4. The frequency of the system.

5. The kind of line-three-phase or single-phase.

6 . The length of the line.

7. The size of the wires.

8. The material of the wires. 
9. The temperature of the wires.

10. The arrangement of the wires on the poles; particularly their distance apart.

The interrelation of these various factors will be considered in detail.

Resistance, Reactance and Capacity, Susceptance and LEAKAGE

For wires of the size ordinarily used in practise and for any frequency up to 60 cycles, the resistance of a solid wire per unit length depends solely upon the material of the wire and its cross section. A stranded cable has a slightly greater resistance than a solid wire, due to the spiralling of the individual wires which made up the cable. In the table on Chart No. 3 are given the resistance and weight per mile of both copper and aluminum for the various sizes between a No. 16 and 1,000,000 circular mils. This table is calculated for a temperature of 20 deg. cent. ( $=68 \mathrm{deg}$. fahr.), assuming a temperature coefficient of 0.42 per cent per degree centigrade; the conductivity of copper is taken as 98 per cent and the conductivity of aluminum as 62 per cent of Matthiessen': standard; and the resistance and the weight of the stranded wires are taken one per cent greater than for solid wire of the same cross section.

The reactance of a wire in ohms is equal to $2 \pi f L \times 10^{-3}$, where $f$ is the frequency and $L$ the self-induction in millihenrys. The self-induction of a round solid wire of radius $a$ inches when the return wire is parallel to it and at a distance $D$ inches from it (center to center) is

$$
L=0.7411 \log _{10} \frac{D}{a}+0.0805 \text { millihenrys per mile. }
$$

This formula applies also when the return circuit consists of two wires each of which is at a distance $D$ from the wire in question; that is, the above formula gives the inductance per mile of each wire whether the system is single-phase or three-phase, provided in the latter case the wires are arranged symmetrically (i.e., form the three edges of an equilateral prism). The inductance of a stranded wire is practically equal to that of a solid wire of the same cross section of conducting material; for a given number on the B. \& S. gage or for a given area in circular mils the inductance is therefore independent of whether the wire is solid or stranded. 
The value of the inductance per mile of wire for various sizes of wire and for various spacings is given on Chart No. 3. This particular form of chart arises from the fact that the inductance depends only upon the ratio $\frac{D}{a}$; consequently, all combinations of sizes and spacings for which this ratio is constant give the same value of the inductance. The reactance for both 25 and 60 cycles is also given on Chart No. 3 .

The capacity susceptance of a wire in ohms is equal to $2 \pi f C$ $\times 10^{-6}$, where $f$ is the frequency and $C$ the capacity in microfarads of the wire to neutral; the susceptance of a wire is the ratio of the charging current to the volts to neutral. The capacity to neutral of a round wire of radius $a$ inches when the return wire is parallel to it and at a distance $D$ inches from it (center to center) is

$$
C=\log _{10} \frac{D}{a}
$$

provided $\frac{D}{a}$ is greater than $12^{*}$ and the distance from the wire to all other conductors is large compared with $D$. This formula applies also when the return conductor consists of two wires each of which is at a distance $D$ from the wire in question. That is, the above formula gives the capacity per mile of each wire whether the system is single-phase or three-phase, provided the wires are at a great distance, compared with $D$, from all other conductors (i.e., overhead lines) and provided that in the case of a three-phase system the wires are arranged symmetrically (i.e., from the three edges of an equilateral prism.) The capacity of a stranded wire is greater than that of a solid wire, since its radius is greater. The above formula is not strictly applicable to a stranded wire, since the latter is not round, but as a first approximation it may be used.

In Chart No. 3 is given the capacity of the various sizes of

${ }^{*}$ When $\frac{D}{a}<12$, the exact formula for the capacity, when the two wires are at a great distance from all other conductors, is

$$
C=\frac{0.08941}{\cosh ^{-1} \frac{D}{2 a}}
$$


wire on various spacings. In calculating the capacity scale the diameter of the wire in each case was taken 15 per cent greater than the diameter of a solid wire of the same cross section; the ratio of the diameter of a stranded wire to a solid wire is approximately 1.15 . The proper correction to obtain the capacity of a solid wire is indicated on the chart. The chart also gives the capacity susceptance at 25 and 60 cycles.

The leakage current between wires under ordinary working conditions is negligible. The leakage need be taken into account only in the case the voltage is sufficiently high to produce the so-called "corona effect". This effect appears only when extra high voltages are used and the wires are comparatively small.

The Equations of a Transmission Line.

Fig. 4 is a diagrammatic representation of a section of a transmission line. Each wire of the line, whether single- or three-

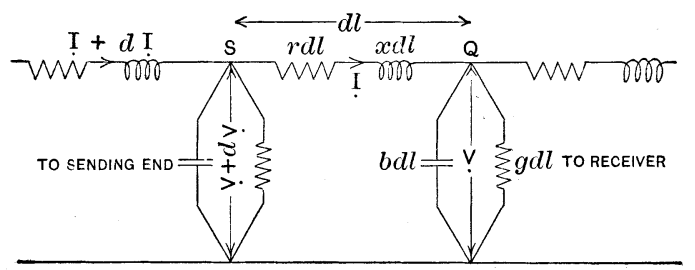

FIG. 4

phase, may be considered separately, and the return for each wire may be represented by a fictitious neutral of zero resistance and zero reactance. Let

$P=$ average watts transmitted past the point $Q$ by all of the wires.

$E=$ effective volts between wires at any point $Q$.

$V=$ effective volts between any one of the line wires and neutral at the point $Q$. (For a single-phase line $V=\frac{E}{2} ;$ for a three-phase line $\left.V=\frac{E}{\sqrt{3}}\right)$

$\varphi=$ power factor angle at $Q$ ( $\varphi$ taken positive for lagging current).

$I=$ effective amperes at the point $Q$. (For a single-phase line $I=\frac{P}{E \cos \varphi}$; for a three-phase line $\left.I=\frac{P}{\sqrt{3} E \cos \varphi}\right)$ 
The same symbols with the subscript " 1 " refer to the receiver. The same symbols with the subscript " 0 " refer to the sending end.

$l=$ distance in miles of this point $Q$ from receiver.

$d l=$ elementary length of line between $Q$ and $S$.

$l_{0}=$ length of line in miles.

$r=$ resistance of wire in ohms per mile.

$x=$ reactance of wire in ohms per mile.

$g=$ leakage conductance, one wire to neutral in mhos per mile.

$b=$ capacity susceptance, one wire to neutral, in mhos per mile.

$$
\begin{aligned}
& z=\sqrt{r^{2}+x^{2}} \\
& y=\sqrt{g^{2}+b^{2}} \\
& \epsilon=\tan ^{-1} \frac{x}{r} \\
& \eta=\tan ^{-1} \frac{b}{g}
\end{aligned}
$$

For the voltage and current sine functions of the time we may use the symbolic method. Let $V$ and $I$ be the symbolic expressions for the voltage and current respectively. The symbolic expression for the impedance per unit length of line is

$$
z=z(\cos \epsilon+j \sin \epsilon)=z e^{j \epsilon}
$$

and the symbolic expression for the admittance of the leakage circuit per mile of line is

$$
y=y(\cos \eta+j \sin \eta)=y e^{j \eta}
$$

Applying Kirchoff's laws to the element $d l$ of the line, we get

$$
\begin{aligned}
& \frac{d V}{d \dot{l}}=z I \\
& \frac{d I}{d \dot{l}}=y V
\end{aligned}
$$

Differentiating (3) with respect to $l$ and substituting the value of $\frac{d I}{d l}$ from (4) gives

$$
\frac{d^{2} V}{d \dot{l^{2}}}=y z \underline{V}
$$


The integral of this equation is

$$
V=A \sinh (\sqrt{y z} l+\delta)
$$

where $A$ and $\delta$ are constants of integration.

Substituting (6) in (4), and integrating with respect to $l$, we get

$$
I=\sqrt{\frac{\bar{y}}{z}} A \cosh (\sqrt{y \underline{z}} l+\delta)
$$

Both $A$ and $\delta$ may be complex; let their symbolic expressions be

$$
\begin{gathered}
A=A(\cos \alpha+j \sin \alpha)=A e^{j \alpha} \\
\delta=\beta+j \gamma
\end{gathered}
$$

From (1) and (2)

$$
\begin{aligned}
& \sqrt{y z}=\sqrt{y z e^{j\left(\frac{\eta+\varepsilon}{2}\right)}}=1^{\prime} \overline{y z}\left[\cos \left(\frac{\eta+\epsilon}{2}\right)+j \sin \left(\frac{\eta+\epsilon}{2}\right)\right] \\
& \sqrt{\frac{\ddot{y}}{z}}=\sqrt{\frac{y}{z}} e^{j\left(\frac{\eta-\varepsilon}{2}\right)}
\end{aligned}
$$

Put

$$
\begin{gathered}
\lambda=\frac{\eta-\epsilon}{2} \\
m=\sqrt{y z} \cos \left(\frac{\eta+\epsilon}{2}\right) \\
n=\sqrt{y z} \sin \left(\frac{\eta+\epsilon}{2}\right)
\end{gathered}
$$

Then (6) and (7) become

$$
\begin{gathered}
\gamma=A \sinh [(\beta+m l)+i(\gamma+n l)] \\
I=\sqrt{\frac{y}{z}} A \cosh [(\beta+m l)+i(\gamma+n l)]
\end{gathered}
$$


But

where

$$
\begin{gathered}
\sinh [(\beta+m l)+j(\gamma+n l)] \\
=\sinh (\beta+m l) \cos (\gamma+n l)+j \cosh (\beta+m l) \sin (\gamma+m l) \\
=M e^{j \mu}
\end{gathered}
$$

$$
M=\sqrt{ } \sinh ^{2}(\beta+m l) \cos ^{2}(\gamma+n l)+\cosh ^{2}(\beta+m l) \sin ^{2}(\gamma+n l)
$$

and

$$
\tan \mu=\frac{\tan (\gamma+n l)}{\tanh (\beta+m l)}
$$

Making use of the relations that, for any argument $u$,

$$
\begin{aligned}
& \sinh ^{2} u=\frac{\cosh 2 u-1}{2} \\
& \cosh ^{2} u=\frac{\cosh 2 u+1}{2} \\
& \sin ^{2} u=\frac{1-\cos 2 u}{2} \\
& \cos ^{2} u=\frac{1+\cos 2 u}{2}
\end{aligned}
$$

the above expression for $M$ may be written

$$
M=\frac{1}{\sqrt{2}} \sqrt{\cosh 2(\beta+m l)-\cos 2(\gamma+n l)}
$$

Whence, substituting for $A$ its value from (8) and the relation (17), the expression (15) for the voltage at any point along the line becomes

$$
V=A M e^{j(\alpha+\mu)}
$$

In an exactly similar manner, we may write

$$
\begin{gathered}
\cosh [(\beta+m l)+j(\gamma+n l)]=N e^{j v} \\
N=\frac{1}{\sqrt{2}} \sqrt{\cosh 2(\beta+m l)+\cos 2(\gamma+n l)}
\end{gathered}
$$




$$
\tan \nu=\tan (\gamma+n l) \tanh (\beta+m l)
$$

Whence the current at any point along the line is

$$
I=\sqrt{\frac{y}{z}} A N e^{j(\alpha+v+\lambda)}
$$

Hence the effective value of the voltage at any point is $A M$ or

$$
V=A_{0} \sqrt{\cosh 2(\beta+m l)-\cos 2(\gamma+n l)}
$$

and the effective value of the current at this point is

$$
I=\sqrt{\frac{\bar{y}}{z}} A_{0} \sqrt{\cosh 2(\beta+m l)+\cos 2(\gamma+n l)}
$$

where $A_{0}$, which is a constant yet to be determined, is put for $\frac{A}{\sqrt{2}}$. The angle by which the current lags behind the voltage, or the power factor angle $\varphi$, is

$$
\begin{gathered}
\varphi=\alpha+\mu-(\alpha+\nu+\lambda) \\
=\mu-\nu-\lambda
\end{gathered}
$$

A simple expression for the angle $(\mu-\nu)$ may be derived by making use of the trigonometric relation

$$
\tan (\mu-\nu)=\frac{\tan \mu-\tan \nu}{1+\tan \mu \tan \nu}
$$

for, substituting for $\tan \mu$ and $\tan \nu$ their values from (17) and (21), we get

$$
\tan (\mu-\nu)=\frac{\sin 2(\gamma+n l)}{\sinh 2(\beta+m l)}
$$

Whence the power factor angle at any point along the line is

$$
\varphi=\tan ^{-\prime}\left[\frac{\sin 2(\gamma+n l)}{\sinh 2(\beta+m l)}\right]-\lambda
$$


The power per phase at any point is then

$$
\begin{gathered}
P^{\prime}=V I \cos \varphi \\
=\sqrt{\frac{y}{z}} A_{0}^{2} \cos \varphi \sqrt{\cosh ^{2} 2(\beta+m l)-\cos ^{2} 2(\gamma+n l)}
\end{gathered}
$$

Making use of the relations

$$
\begin{aligned}
\cosh ^{2} u & =1+\sinh ^{2} u \\
\cos ^{2} u & =1-\sin ^{2} u
\end{aligned}
$$

we may write this expression

$$
P^{\prime}=\sqrt{\frac{y}{z}} A_{0}^{2} \cos \varphi \sqrt{\sinh ^{2} 2}(\beta+m l)+\sin ^{2} 2(\gamma+n l)
$$

From (25), making use of the trigonometric formula for the cosine of the difference of two angles,

$$
\cos \varphi=\frac{\sinh 2(\beta+m l) \cos \lambda+\sin 2(\gamma+n l) \sin \lambda}{\sqrt{\sinh ^{2} 2(\beta+m l)+\sin ^{2} 2(\gamma+n l)}}
$$

Whence

$$
P^{\prime}=\sqrt{\frac{y}{z}} A_{0}{ }^{2}[\sinh 2(\beta+m l) \cos \lambda+\sin 2(\gamma+m l) \sin \lambda](26)
$$

At the receiver $l=0$; and at the sending end $l=l_{0}$. Hence, from (23), we have that the ratio of the volts $E_{0}$ at the sending end to the volts $E_{1}$ at the receiver is

$$
\frac{E_{0}}{E_{1}}=\frac{V_{0}}{V_{1}}=\sqrt{\frac{\cosh 2\left(\dot{\beta}+m l_{0}\right)-\cos 2\left(\gamma+n l_{0}\right)}{\cosh 2 \beta-\cos 2 \gamma}}
$$

From (24) the ratio of the current $I_{0}$ at the sending end to current $I_{1}$ at the receiver is

$$
\frac{I_{0}}{I_{1}}=\sqrt{\frac{\cosh 2\left(\beta+m l_{0}\right)+\cos 2\left(\gamma+n l_{0}\right)}{\cosh 2 \beta+\cos 2 \gamma}}
$$

From (26) the ratio of the power $P_{0}$ at the sending end to the power $P_{1}$ at the receiver is

$$
\frac{P_{0}}{P_{1}}=\frac{P_{0}^{\prime}}{P_{1}^{\prime}}=\frac{\sinh 2\left(\beta+m l_{0}\right) \cos \lambda+\sin 2\left(\gamma+n l_{0}\right) \sin \lambda}{\sinh 2 \beta \cos \lambda+\sin 2 \gamma \sin \lambda}
$$


We have now to determine the two constants $\beta$ and $\gamma$. From (25), putting $l=0$, we have

$$
\frac{\sin 2 \gamma}{\sinh 2 \beta}=\tan \left(\varphi_{1}+\lambda\right)
$$

and from (23) and (24), putting $l=0$, and squaring, we have

$$
\begin{gathered}
V_{1}^{2}=A_{0}^{2}(\cosh 2 \beta-\cos 2 \gamma) \\
\frac{z}{y} I_{1}^{2}=A_{0}^{2}(\cosh 2 \beta+\cos 2 \gamma)
\end{gathered}
$$

Adding and subtracting these two expressions and taking the ratio of the results gives

$$
\frac{\cosh 2 \beta}{\cos 2 \gamma}=\frac{z I_{1}^{2}+y V_{1}^{2}}{z I_{1}^{2}-y V_{1}^{2}}
$$

Put

$$
\rho=\frac{z l_{0} I_{1}}{V_{1}}=\text { ratio of total line impedance volts to volts de- }
$$
livered.

$$
\sigma=\frac{y l_{0} V_{1}}{I_{1}}=\text { ratio of total line admittance current to current }
$$
delivered.

Then

$$
\frac{z I_{1}^{2}+y V_{1}^{2}}{z I_{1}^{2}-y V_{1}^{2}}=\frac{\rho+\sigma}{\rho-\sigma}
$$

whence

$$
\cosh 2 \beta=\frac{\rho+\sigma}{\rho-\sigma} \cos 2 \gamma
$$

From (30)

$$
\sinh 2 \beta=\frac{\sin 2 \gamma}{\tan \left(\varphi_{1}+\lambda\right)}
$$

Squaring and subtracting we get

$$
1=\left(\frac{\rho+\sigma}{\rho-\sigma}\right)^{2} \cos ^{2} 2 \gamma-\begin{gathered}
\sin ^{2} 2 \gamma \\
\tan ^{2}\left(\varphi_{1}+\lambda\right)
\end{gathered}
$$


Making use of the trigonometric relations

$$
\begin{aligned}
& \cos ^{2} 2 \gamma=\frac{1}{1+\tan ^{2} 2 \gamma} \\
& \sin ^{2} 2 \gamma=\frac{\tan ^{2} 2 \gamma}{1+\tan ^{2} 2 \gamma}
\end{aligned}
$$

and solving for $\tan 2 \gamma$, we get

$$
\tan 2 \gamma=\frac{2 \sqrt{\rho \sigma} \sin \left(\varphi_{1}+\lambda\right)}{\rho-\sigma}
$$

Note that, from (31) and (31a), $\sin 2 \gamma$ and $\cos 2 \gamma$ have respectively the same algebraic signs as the numerator and denominator of this fraction. This is important since the algebraic sign of the tangent alone does not fix the quadrant in which an angle lies. The value of $2 \gamma$ may be taken directly from the trigonometric scale on Chart No. 3. (If the second member of (32) is greater than unity, use the reciprocal which is equal to the $\cot 2 \gamma$ ). From (30) we then have

$$
\sinh 2 \beta=\frac{\sin 2 \gamma}{\tan \left(\varphi_{1}+\lambda\right)}
$$

from which $2 \beta$ may be obtained from Chart No. 5.

Approximate Equations for Ordinary Lines.

In case the length of the line does not exceed 100 miles when the frequency is 60 cycles or 200 miles when the frequency is 25 cycles the above equations may be greatly simplified by making use of an approximation which will not introduce an appreciable error in any case likely to arise in practise.

The simplification results from the following substitutions in equations (27) to (29)

$$
\begin{gathered}
\cosh 2 m l=1+2 m^{2} l^{2} \\
\sinh 2 m l=2 m l \\
\cos 2 n l=1-2 n^{2} l^{2} \\
\sin 2 n l=2 n l
\end{gathered}
$$


where $n l$ is expressed in radians. These approximations are accurate to within 2 per cent for $2 \mathrm{ml}$ less than 0.35 and for $2 n l$ less than 0.35 radians or $20 \mathrm{deg}$.

From equations (31), (31a) and (32) we have

$$
\sinh 2 \beta=\frac{2 \sqrt{\rho \sigma} \cos \left(\varphi_{1}+\lambda\right) \cos 2 \gamma}{\rho-\sigma}
$$

$$
\cosh 2 \beta=\frac{\rho+\sigma}{\rho-\sigma} \cos 2 \gamma
$$

$$
\sin 2 \gamma=\frac{2 \sqrt{\rho \sigma} \sin \left(\varphi_{1}+\lambda\right) \cos 2 \gamma}{\rho-\sigma}
$$

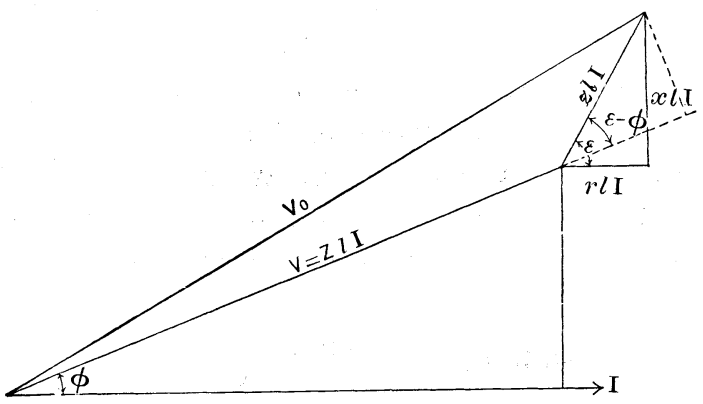

FIG. 5

Substituting equations (34) to (37) in (27) to (29) we get, for no leakage,

$$
\begin{gathered}
\frac{E_{0}}{E}=\sqrt{1+2 \rho \cos \left(\epsilon-\varphi_{1}\right)+\rho^{2}-b x l_{0}^{2}} \\
\frac{I_{0}}{I}=\sqrt{1+2 \sigma \cos \left(90-\varphi_{1}\right)+\sigma^{2}-b x l_{0}^{2}} \\
\frac{P_{0}}{P}=1+\frac{\rho \cos \epsilon}{\cos \varphi}-b r l_{0}^{2} \tan \varphi_{1}
\end{gathered}
$$

It is interesting to note that these last three equations, when $b$ is put equal to zero, are identical with the relations which follow immediately from the vector diagram, Fig. 5, of the line and load when the capacity of the line is neglected. 


\section{SUMMARY}

In the working formulas given below, the symbols for voltage, current, etc., at the receiver will be written without the subscripts, and $l$ will be used to designate the length of the line from receiver to sending end. The power will also be expressed in kilowatts. The leakage will be assumed zero.

$P=$ kilowatts delivered to receiver.

$E=$ volts between wires at receiver.

$K=$ power factor of receiver.

$\varphi=\cos ^{-1} K=$ power factor angle of receiver (see Chart No. 3.)

$I=$ amperes taken by receiver. (For a three-phase line

$$
\left.I=\frac{P \times 1000}{\sqrt{3 E K}} ; \text { for a single-phase line } I=\frac{P \times 1000}{E K}\right) .
$$

The same symbols with the subscript zero refer to the sending end.

$l=$ distance of transmission in miles.

$r=$ resistance of wire in ohms per mile (see Chart No. 3).

$x=$ reactance of wire in ohms per mile (see Chart No. 3).

$b=$ capacity susceptance, one wire to neutral, in mhos per mile. (See Chart No. 3).

$\boldsymbol{\epsilon}=\tan ^{-\prime} \frac{x}{r}=$ power factor angle of the line (see Chart No. 3).

$Z=$ equivalent impedance of receiver terminal to neutral, per mile of line.

$=\frac{E}{\sqrt{3} l I}$ for a three-phase line;

$=\frac{E}{2 l I}$ for a single-phase line.

Exact Method. Since the leakage is assumed zero, the admittance $y$ is equal to $b$ and the power factor angle of the leakage circuit is $90 \mathrm{deg}$. Hence to determine the voltage, current, etc., at the sending end proceed as follows:

First calculate the constants

$$
\begin{gathered}
\lambda=\frac{90-\epsilon}{2} \\
u=2 l \sqrt{\frac{b x}{\sin \epsilon}} \sin \lambda
\end{gathered}
$$




$$
0=114.6 l \sqrt{\frac{b x}{\sin \epsilon}} \cos \lambda
$$

$d=\frac{100 r}{Z \cos \epsilon}=$ per cent ratio of impedance volts to volts delivered

$d^{\prime}=100 b Z l^{2}=$ per cent ratio of charging current to current delivered.

$$
0_{0}=\tan ^{-\prime}\left[\frac{2 \sqrt{d d^{\prime}} \sin (\varphi+\lambda)}{d-d^{\prime}}\right]
$$

The algebraic signs of $\sin \theta_{0}$ and $\cos \theta_{0}$ are respectively the same as the numerator and denominator of this fraction; this fixes the quadrant in which $\theta_{0}$ lies.

$$
u_{0}=\sinh ^{-\prime}\left[\frac{\sin \theta_{0}}{\tan (\varphi+\lambda)}\right]
$$

Then

$$
\begin{gathered}
\frac{E_{0}}{E}=\sqrt{\frac{\cosh \left(u_{0}+u\right)-\cos \left(\theta_{0}+\theta\right)}{\cosh u_{0}-\cos \theta_{0}}} \\
\frac{I_{0}}{I}=\sqrt{\frac{\cosh \left(u_{0}+u\right)+\cos \left(\theta_{0}+\theta\right)}{\cosh u_{0}+\cos \theta_{0}}} \\
\frac{P_{0}}{P}=\frac{\sinh \left(u_{0}+u\right) \cos \lambda+\sin \left(\theta_{0}+\theta\right) \sin \lambda}{\sinh u_{0} \cos \lambda+\sin \theta_{0} \sin \lambda}
\end{gathered}
$$

The power factor angle $\varphi$ at the sending end may also be determined directly from the formula

$$
\varphi_{0}=\tan ^{-\prime}\left[\frac{\sin \left(\theta_{0}+\theta\right)}{\sinh \left(u_{0}+u\right)}\right]-\lambda
$$

An excellent check on the results obtained is to compare the value of $\cos \varphi_{0}$ determined from this formula with the value of the power factor calculated from the voltage $E_{0}$, cur- 
rent $I_{0}$, and power $P_{0}$ determined from the three preceding formulas. (For a three-phase line $\cos \varphi_{0}=\frac{1000 P_{0}}{\sqrt{3} E_{0} I_{0}}$; for a single-phase line $\left.\cos \varphi_{0}=\begin{array}{c}1000 P_{0} \\ E_{0} I_{0}\end{array}\right)$.

Approximate Method, Neglecting Line Capacity. In the case of a short line (i.e., $10^{4} b \times l^{2}$ negligible compared with $d$ ) the relations between the voltage, current and power at the two ends of the line are as follows. Let

$p=100 \frac{E_{0}-E}{E}=$ the per cent ratio of the difference in volts

at the two ends to the volts delivered, i.e., the per cent "volts drop".

$q=100 \frac{P_{0}-P}{P}=$ the per cent ratio of power lost in line to

Then power delivered, i.e., the per cent " power loss".

$$
\begin{gathered}
p=\sqrt{ } 10^{4}+200 d \cos (\epsilon-\varphi)+d^{2}-100 \\
q=\frac{d \cos \epsilon}{K}=\frac{100 r}{K Z} \\
K_{0}=\frac{100+q}{100+p} \cdot K
\end{gathered}
$$

Chart No. 4 gives a graphical solution of equation (52). The ordinate scale of this chart gives directly the value of the per cent volts drop when the per cent impedance drop $d$ and the difference in the power factor angles of the line and load are known. The method of using the chart is described in detail on the chart itself. Each of the curves on the chart was drawn by plotting the values of

$$
200 d \cos (\epsilon-\varphi)+d^{2}
$$

as actual vertical distance against $d$ as abscissas; the numbers on the curves give the corresponding values of $(\epsilon-\varphi)$. The scale of ordinates marked on the left is a square root scale, the numbers giving the value of 
Note that since $\cos (\epsilon-\varphi)=\cos (\varphi-\epsilon)$, the algebraic sign of the resultant angle $(\epsilon-\varphi)$ is immaterial. The curve marked with a given number, say 50 , is to be used whether $\epsilon-\varphi=+50$ or $\epsilon-\varphi=-50$.

Approximate Method when Line Capacity is Taken into Account. For longer lines (i.e., when $10^{4} b x l^{2}$ is not negligible in comparison with $d$ ), the following close approximations hold, provided the length of the line is not over 100 miles for 60 cycles or over 200 miles for 25 cycles. Let

$p^{\prime}=$ true value of the per cent volts drop when corrected for capacity effect.

$q^{\prime}=$ true value of the per cent power loss when corrected for capacity effect.

$p^{\prime}{ }_{i}=100 \frac{I_{0}-I}{I}=$ the per cent ratio of the difference in the current at the two ends of the line to the current delivered; i.e., the per cent "current loss" $\left(p_{i}\right.$ will in general be negative for a lagging current at the receiver).

Then

$$
\begin{gathered}
p^{\prime}=\sqrt{10^{4}+200 d \cos (\epsilon-\varphi)+d^{2}-10^{4} b \times l^{2}-100} \\
p_{i}{ }^{\prime}=\sqrt{10^{4}+200 d^{\prime} \cos (90+\varphi)+\left(d^{\prime}\right)^{2}-10^{4} b x l^{2}-100} \\
q^{\prime}=q-100 b r l^{2} \tan \varphi \\
K_{0}{ }^{\prime}=c\left(100+q^{\prime}\right) K \\
\left(100+p^{\prime}\right)\left(100+p^{\prime}{ }_{i}\right)
\end{gathered}
$$

Equations (55) and (56) may be solved graphically by the use of Chart No. 4. Distances on the " capacity correction scale" in the upper left hand corner of this chart are laid off equal to $10^{4} b \times l^{2}$, the number on this scale giving the corresponding value of $b \times l^{2}$. The method of making this correction is described on the chart itself.

Chart No. 4 may also be used to calculate the regulation in any case where the voltage drop is due to the impedance of the apparatus. For example, it may be used to calculate the regulation of a transformer when $\epsilon$ is taken equal to the power factor angle of the equivalent impedance of the transformer and $d$ is taken equal to the per cent ratio of the equivalent impedance of 
the transformer to the equivalent impedance $(V / I)$ of the load, or to calculate the regulation of a generator when $\epsilon$ is taken equal to the power factor angle of the equivalent impedance of the generator and $d$ is taken equal to the per cent ratio of the synchronous impedance of the generator to the impedance of the load $(V / I)$.

\section{EXAMPLE}

Given the same example as noted on Chart No. 4, namely:

$$
\begin{aligned}
& E=60,000 \text { volts } \quad l=100 \text { miles } \quad b=6.03 \times 10^{-6} \mathrm{ohms} \\
& I=100 \text { amperes } \quad r=0.267 \mathrm{ohms} \quad \epsilon=69.83 \mathrm{deg} \text {. } \\
& K=0.95 \mathrm{lag} \quad x=0.727 \mathrm{ohms} \quad \cos \epsilon=0.3447 \\
& \text { Then, } Z=10.083 \\
& d=22.36 \\
& \lambda=10.08 \quad d^{\prime}=20.89 \\
& u=0.07567 \quad \theta_{0}=85.89 \\
& \theta=24.38 \quad u_{0}=1.378
\end{aligned}
$$

Whence,

$$
\begin{array}{ll}
\text { Exact method } & \begin{array}{c}
\text { Approximate method } \\
\text { (See Chart No. } 4)
\end{array} \\
p^{\prime \prime}=13.01 \text { per cent } & p^{\prime}=13.6 \text { per cent } \\
q^{\prime \prime}=6.43 \text { per cent } & q^{\prime}=-6.7 \text { per cent } \\
K^{\prime \prime}{ }_{0}=96.68 & K_{0}^{\prime}=96.5
\end{array}
$$

